\title{
MORFOMETRÍA DE ESPECIES DE TRICHOGRAMMA WESTWOOD (HYMENOPTERA: TRICHOGRAMMATIDAE) DE CENTROS REPRODUCTORES DE MÉXICO
}

\author{
Fabián García-GonZÁLEZ1, Alejandro GonZÁLEZ-HERNÁNDEZ², \\ Víctor Manuel PINTO ${ }^{3}$ y Samuel RAMírez-AlarCón ${ }^{3}$ \\ 1 Unidad Regional Universitaria de Zonas Áridas, Universidad Autónoma Chapingo. Carr. Gómez \\ Palacio-Cd Juárez. Domicilio Conocido, Bermejillo, Durango. MÉXICO. CP 35230. \\ e-mail: fabiangglez@yahoo.com \\ ${ }^{2}$ Facultad de Ciencias Biológicas. Universidad Autónoma de Nuevo León. Ave Pedro de Alba s/n, \\ San Nicolás de los Garza, N. L. MÉXICO. CP 66400. e-mail: agonzale@fcb.uanl.mx \\ 3Posgrado en Protección Vegetal, Depto. de Parasitología Agrícola, Universidad Autónoma Chapingo, \\ Carr. México-Texcoco Km. 38.5. Chapingo, Edo. de Méx. MÉXICO. CP 5623. \\ Autor para correspondencia: vmpinto@correo.chapingo.mx
}

García-González, F., A. González-Hernández, V. M. Pinto y S. Ramírez-Alarcón. 2009. Morfometría de especies de Trichogramma Westwood (Hymenoptera: Trichogrammatidae) de centros reproductores de México. Acta Zoológica Mexicana (n. s.), 25(2): 409-425.

RESUMEN. La importancia de Trichogramma como agente de control biológico de plagas contrasta con el conocimiento de su taxonomía en México, debido a que no existen estudios que muestren una adecuada identidad de las especies que se reproducen y existen en forma nativa en las áreas agrícolas. El objetivo de este estudio fue realizar un análisis morfométrico de las especies de Trichogramma reproducidas masivamente en México como técnica de corroboración de determinación de especies realizada en base a su morfología. El análisis de varianza y la comparación de medias por el método de Duncan permitieron separar a T. pretiosum y $T$. exiguum de $T$. fuentesi y $T$. pintoi con la longitud de la seta funicular más larga de la antena del macho. Con la longitud del proceso intervoselar de las genitalias de los machos fue posible diferenciar a $T$. pretiosum y $T$. pintoi de $T$. fuentesi y T. exiguum. En el análisis de grupos (cluster jerárquico), la longitud del proceso intervoselar de la genitalia permitió diferenciar las cuatro especies referidas.

Palabras clave: Control biológico, genitalia, parasitoides, proceso intervoselar, seta funicular, taxonomía.

García-González, F., A. González-Hernández, V. M. Pinto, \& S. Ramírez-Alarcón. 2009. Morphometry of Trichogramma Westwood (Hymenoptera: Trichogrammatidae) species from mass rearing centers of Mexico. Acta Zoológica Mexicana (n. s.), 25(2): 409-425.

ABSTRACT. The importance of Trichogramma, as a biological control agent of crop pests, contrasts with the knowledge of his taxonomy in Mexico, due to the lack of studies that show a suitable identity of the species that are mass reared and exist in native form in agricultural areas. The objective of this

Recibido: 15/01/2009; aceptado: 29/05/2009. 
study was made a morphometric analysis of the Trichogramma species mass rearing in Mexico as a corroborated technique of determination based in morphology. The analysis of variance and mean comparisons by the Duncan's test allowed separate T. pretiosum and T. exiguum from T. fuentesi and T. pintoi based on the longest funicular setae length of male antenna. With the intervoselar process length of male genitalia, it was possible to differentiate $T$. pretiosum and $T$. pintoi from $T$. fuentesi and $T$. exiguum. In the cluster analysis (hierarchic cluster analysis), the intervoselar process length allowed differentiate the four species referred.

Key words: Biological control, genitalia, parasitoids, intervoselar process, funicular setae, taxonomy.

\section{INTRODUCCIÓN}

De los 83 géneros que tiene la familia Trichogrammatidae (Pinto, 1998; Owen y Pinto, 2004; Pinto y George, 2004; Pinto y Owen, 2004), Trichogramma es el más importante y ha sido ampliamente usado como agente de control biológico desde hace cerca de 100 años. No obstante este género fue considerado seriamente para el control de plagas, principalmente de lepidópteros, hasta que Flanders (1930) desarrolló la técnica de su reproducción masiva. En México, actualmente se conoce una rica y diversa fauna de tricogramátidos, considerando que en el país confluyen dos regiones zoogeográficas bien representadas: la región Neártica y la región Neotropical. Lo anterior concuerda con la alta riqueza y diversidad de otros grupos de Chalcidoidea registrada en México (González, 2000). El origen de esta diversidad de tricogramátidos en México puede estar relacionada al gran parecido que tienen las especies recientes con las encontradas en ámbar de oligoceno en Chiapas, México, y es probable que el hábitat base de desarrollo en los huevos de otros insectos es de al menos 30 millones de años de edad (Doutt y Viggiani, 1968).

La práctica de la identificación de especies de Trichogramma, es importante ya que permite el conocimiento de la diversidad especies de este grupo de organismos y permite elaborar estudios relativos a su conservación y aprovechamiento sustentable, aunque como la mayoría de los micro himenópteros, los tricogramátidos a menudo pasan desapercibidos para los colectores por su tamaño diminuto y en consecuencia son relativamente desconocidos (Pinto, 1997). Aun conociendo los registros que actualmente existen de este parasitoide obtenidos de colectas de campo como de centros reproductores, se considera que en México existe poco conocimiento de las especies de Trichogramma. También es posible que entre el personal involucrado con este parasitoide no se tenga un conocimiento sobre la taxonomía de este micro himenóptero ya que al revisar los trabajos relativos al mismo, presentados en ocho reuniones y once congresos nacionales de control biológico de 1976 a 2001, se encontró que solo el 15\% (14 estudios) indican las especies de tricógramas y el $85 \%$ (78 estudios) no, refiriéndose solo a Trichogramma, Trichogramma sp, o Trichogramma spp. Esto refleja la necesidad de realizar la presente investigación para profundizar en el conocimiento de la taxonomía de 
este grupo con la finalidad de contribuir técnicamente en la realización de proyectos de investigación y aplicados en México.

\section{MATERIAL Y MÉTODOS}

Se revisaron 305 especímenes de machos de este micro himenóptero, montados en un mismo número de laminillas en bálsamo de Canadá, de los cuales 217 pertenecieron a $T$. pretiosum, 64 a $T$. fuentesi, 14 a $T$. exiguum y 10 a $T$. pintoi. De las laminillas fueron tomadas 2,071 fotografías de siete estructuras de la genitalia que fueron: longitud de la cápsula genital (LCG), ancho de la cápsula genital (ACG), longitud del edeago (LED), longitud del proceso intervoselar (LPIV), longitud de la extensión posterior de la lamina dorsal (LEPLD, ancho de la lamina dorsal (ALD y longitud de la apertura dorsal (LAPD); dos de la antena: longitud de la seta funicular (LSF) y longitud de la antena (LA); cinco del ala anterior: longitud de la vena estigmal (LGVS), ancho de la vena estigmal (ANVS), longitud de la seta marginal (LSM), longitud del ala anterior (LAA) y ancho del ala anterior (AAA); y tres del ala posterior: longitud de la seta marginal (LSMA), longitud (LGAP) y ancho (ANAP), utilizando una cámara Nikon D100 y un microscopio Carl Zeiss ${ }^{\circledR}$ modelo Axiostar Plus con objetivos 10x y 40x CP achromat de óptica infinita. Una vez obtenidas las fotografías, se realizó la medición de las estructuras con el software Image Pro ${ }^{\circledR}$ Plus Ver. 4.0. de la empresa Media Cybernetics (Anónimo, 1999a).

Las especies antes referidas fueron determinadas previamente en base a características morfológicas utilizando las claves de Pinto (1998), Pinto et al. (1983 y 1986), y fueron obtenidas de 23 muestras de 17 centros reproductores y comercializadores de México, distribuidos en los estados de Coahuila (Torreón 2 centros), Colima (Villa de Alvares), Chihuahua (Cuauhtémoc), Guanajuato (Irapuato), Jalisco (Zapotlanejo), Michoacán (Uruapan), Morelos (Cuernavaca), Nuevo León (General Terán), Sinaloa (Los Mochis, Culiacán y Guasave), Sonora (Hermosillo, Cd. Obregón y Navojoa), Tamaulipas (Matamoros), Veracruz (Veracruz) y Zacatecas (Calera) (Cuadro 1).

En el análisis estadístico de las mediciones realizadas a las partes morfológicas antes referidas, se realizó un análisis de varianza (ANOVA) y la comparación de medias por el método de Duncan (C. M. D.) para caracterizar las especies antes citadas, en tanto que para realizar una agrupación de los centros reproductores de cada especie se llevó a cabo un análisis de grupos (análisis de cluster jerárquico) con el método de agrupación de la distancia euclidiana al cuadrado. Ambos análisis se realizaron utilizando el software SPSS Inc. 1999 (Anónimo, 1999b). 
Cuadro 1. Especies de Trichogramma de centros reproductores de México por estado.

\begin{tabular}{|c|c|c|c|c|c|}
\hline Centro & Ubicación & T. exiguum & T. fuentesi & T. pintoi & T. pretiosum \\
\hline 1 & Coahuila & & $\mathrm{X}$ & & \\
\hline 2 & Coahuila & $X$ & & & $\mathrm{X}$ \\
\hline M1 & Colima & $X$ & $\mathrm{X}$ & & $X$ \\
\hline $3 \mathrm{M} 2$ & Colima & & & & $\mathrm{X}$ \\
\hline 4 & Chihuahua & & & & $X$ \\
\hline $5 \mathrm{M} 1$ & Guanajuato & & & & $X$ \\
\hline $5 \mathrm{M} 2$ & Guanajuato & & $X$ & $\mathrm{X}$ & \\
\hline 6 & Jalisco & & & & \\
\hline 7 & Michoacán & & & & $X$ \\
\hline 8 & Morelos & & & & $\mathrm{X}$ \\
\hline 9M1 & Nuevo León & & & & $\mathrm{X}$ \\
\hline $9 \mathrm{M} 2$ & Nuevo León & & & & $X$ \\
\hline 10 & Sinaloa & & & & $\mathrm{X}$ \\
\hline 11 & Sinaloa & & & & $\mathrm{X}$ \\
\hline 12 & Sinaloa & & & & $\mathrm{X}$ \\
\hline 13 & Sonora & & & & $\mathrm{X}$ \\
\hline 14 & Sonora & & & & $X$ \\
\hline 15 & Sonora & & & & $X$ \\
\hline 16 & Tamaulipas & & & & $X$ \\
\hline 17 & Veracruz & & & & $X$ \\
\hline 18 & Zacatecas & & $\mathrm{X}$ & & \\
\hline
\end{tabular}

$\mathrm{M}=$ muestra

\section{RESULTADOS}

Del análisis de varianza (ANOVA) y la comparación de medias por el método de Duncan (C. M. D.) realizados a la matriz de datos elaborada para el análisis estadístico, se obtuvo un resumen de resultados indicados en el Cuadro 2, del cual se indicar que según el valor de la $\mathrm{F}$ estimada (67.834) y a la alta significancia ( $\leq$ 0.01) obtenida, la longitud de la seta funicular de la antena (LSF) permitió separar mejor las especies referidas, sin embargo las medias y la C. M. D. reflejaron cierta relación entre $T$. fuentesi y $T$. pintoi, no obstante $T$. fuentesi presentó una mayor desviación estándar lo cual indica una mayor variación de los datos de esta especie que los de $T$. pintoi, además morfológicamente son muy diferentes. T. pretiosum presentó la mayor longitud de esta estructura $(0.075 \mathrm{~mm}$.) en tanto que T. exiguum la menor longitud $(0.042 \mathrm{~mm}$.). 
Otras estructuras que permitieron separar las especies referidas son la longitud del ala posterior (LAP), ancho de la lamina dorsal de la genitalia (ALD) y la longitud del proceso intervoselar (LPIV), las cuales presentaron una alta significancia $(\leq 0.01) \mathrm{y}$ F estimada de $39.639,39.204$ y 29.478 , respectivamente. En contraste, las variables que presentaron resultados no significativos $(>0.05)$ para la separación de las especies son el ancho de la ala anterior (AAA), longitud de la capsula genital (LCG), la longitud de la vena estigmal de la ala anterior (LVE) y el ancho de la ala posterior (AAP) con valores de F estimada de 1.161, 1.362, 2.127 y 2.358, respectivamente.

$\mathrm{Al}$ analizar los datos de las variables de cada estructura diagnosticada (genitalia, antena, ala anterior y ala posterior), se encontró lo siguiente:

Genitalia: Cinco de las siete variables revisadas presentaron datos altamente significativos $(\leq 0.01)$, sin embargo las que presentaron valores de $\mathrm{F}$ más altos, fueron el ancho de la lamina dorsal (ALD), la longitud del proceso intervoselar (LPIV) y la longitud de la extensión posterior de la lamina dorsal (LEPLD), las cuales permiten diferenciar a $T$. pretiosum y $T$. pintoi, sin embargo $T$. fuentesi y $T$. exiguum presentan una estrecha relación según los valores de las medias, desviación estándar y la C. M. D., las cuales tienen una genitalia, muy similar. Aunque estadísticamente no existen diferencias en la LCG, se aprecia en el Cuadro 2 que $T$. exiguum presentó la mayor longitud de esta variable $(0.141 \mathrm{~mm})$. T. exiguum y $T$. fuentesi presentaron una gran similitud como grupo de especies sibilinas en particular en las variables ACG, LPIV, LEPLD y ALD.

Antena: Las dos variables de la antena estudiadas presentaron una alta significancia en sus resultados, no obstante la longitud de la seta funicular (LSF) presentó el valor más alto de la $\mathrm{F}$ estimada. T. pretiosum fue separada de las otras tres especies con una media de $0.075 \mathrm{~mm}$. T. fuentesi y T. exiguum fueron diferenciadas con medias de $0.053 \mathrm{~mm}$ y $0.042 \mathrm{~mm}$, respectivamente, sin embargo en la LAN, son estadísticamente iguales. $T$. pintoi fue muy similar a $T$. fuentesi en esta estructura, sin embargo por sus estructuras restantes, fueron muy diferentes.

Ala anterior: De las cinco variables estudiadas, los resultados de la longitud de la seta marginal (LSM) y el ancho de la vena estigmal (AVE), presentaron una alta significancia estadística $(\leq 0.01)$, no obstante según la comparación de medias por el método de Duncan, la única especie que puede diferenciarse es $T$. pintoi. $T$. pretiosum, T. fuentesi y T. exiguum son estadísticamente similares en estas dos variables y en la longitud de la vena estigmal (LVE). Se puede indicar que las variables del ala anterior no permitieron separar las especies antes referidas.

Ala posterior: Dos de las tres variables analizadas, presentaron una alta significancia estadística, sin embargo la longitud del ala posterior presento el mayor valor de F (39.639) y permitió diferenciar a $T$. pretiosum, T. exiguum y T. pintoi. T. fuentesi presentó una relación con $T$. pretiosum, las cuales morfológicamente son muy diferentes. 
Analizando en conjunto los resultados indicados en el Cuadro 2, y considerando que $T$. fuentesi y $T$. exiguum taxonómicamente forman un grupo hermano y presentan una estrecha relación, la genitalia, en particular la longitud del proceso intervoselar (LPIV) y el ancho de la lamina dorsal (ALD) fueron las estructuras que permitieron diferenciar adecuadamente las especies antes citadas.

Cuadro 2. Media en mm ( \pm desviación estándar) de diez y siete variables morfométricas de cuatro especies de Trichogramma de centros reproductores de México.

\begin{tabular}{|c|c|c|c|c|c|}
\hline Variable & T. pretiosum & T. fuentesi & T. exiguum & T. pintoi & Media \\
\hline \multicolumn{6}{|l|}{ Genitalia } \\
\hline LCG (NS) & $\begin{array}{c}0.135 a \\
( \pm 0.010)\end{array}$ & $\begin{array}{c}0.136 \mathrm{a} \\
( \pm 0.011)\end{array}$ & $\begin{array}{c}0.141 \mathrm{a} \\
( \pm 0.007)\end{array}$ & $\begin{array}{c}0.135 a \\
( \pm 0.007)\end{array}$ & $\begin{array}{c}0.135 \\
( \pm 0.010)\end{array}$ \\
\hline $\mathrm{ACG}^{* *}$ & $\begin{array}{c}0.038 b \\
( \pm 0.004)\end{array}$ & $\begin{array}{c}0.041 b \\
( \pm 0.003)\end{array}$ & $\begin{array}{c}0.041 b \\
( \pm 0.003)\end{array}$ & $\begin{array}{c}0.033 b \\
( \pm 0.002)\end{array}$ & $\begin{array}{c}0.039 \\
( \pm 0.004)\end{array}$ \\
\hline LED** & $\begin{array}{c}0.128 b \\
( \pm 0.009)\end{array}$ & $\begin{array}{c}0.130 \mathrm{~b} \\
( \pm 0.011)\end{array}$ & $\begin{array}{c}0.134 b \\
( \pm 0.007)\end{array}$ & $\begin{array}{c}0.120 \mathrm{a} \\
( \pm 0.004)\end{array}$ & $\begin{array}{c}0.129 \\
( \pm 0.009)\end{array}$ \\
\hline LPIV $^{* *}$ & $\begin{array}{c}0.012 b \\
( \pm 0.003)\end{array}$ & $\begin{array}{c}0.014 \mathrm{c} \\
( \pm 0.002)\end{array}$ & $\begin{array}{c}0.015 \mathrm{c} \\
( \pm 0.001)\end{array}$ & $\begin{array}{c}0.005 \mathrm{a} \\
( \pm 0.001)\end{array}$ & $\begin{array}{c}0.012 \\
( \pm 0.003)\end{array}$ \\
\hline LEPLD** & $\begin{array}{c}0.035 \mathrm{a} \\
( \pm 0.003)\end{array}$ & $\begin{array}{c}0.039 \mathrm{~b} \\
( \pm 0.005)\end{array}$ & $\begin{array}{c}0.038 b \\
( \pm 0.004)\end{array}$ & $\begin{array}{l}0.037 \mathrm{ab} \\
( \pm 0.003)\end{array}$ & $\begin{array}{c}0.036 \\
( \pm 0.004)\end{array}$ \\
\hline ALD** & $\begin{array}{c}0.030 \mathrm{~b} \\
( \pm 0.003)\end{array}$ & $\begin{array}{c}0.034 c \\
( \pm 0.003)\end{array}$ & $\begin{array}{c}0.034 c \\
( \pm 0.003)\end{array}$ & $\begin{array}{c}0.023 a \\
( \pm 0.004)\end{array}$ & $\begin{array}{c}0.031 \\
( \pm 0.004)\end{array}$ \\
\hline LAPD* & $\begin{array}{c}0.079 b \\
( \pm 0.006)\end{array}$ & $\begin{array}{l}0.077 \mathrm{ab} \\
( \pm 0.006)\end{array}$ & $\begin{array}{c}0.081 b \\
( \pm 0.006)\end{array}$ & $\begin{array}{c}0.074 a \\
( \pm 0.004)\end{array}$ & $\begin{array}{c}0.079 \\
( \pm 0.006)\end{array}$ \\
\hline \multicolumn{6}{|l|}{ Antena } \\
\hline $\mathrm{LSF}^{* *}$ & $\begin{array}{c}0.075 \mathrm{c} \\
( \pm 0.010)\end{array}$ & $\begin{array}{c}0.053 b \\
( \pm 0.016)\end{array}$ & $\begin{array}{c}0.042 \mathrm{a} \\
( \pm 0.013)\end{array}$ & $\begin{array}{c}0.054 b \\
( \pm 0.003)\end{array}$ & $\begin{array}{c}0.069 \\
( \pm 0.015)\end{array}$ \\
\hline $\mathrm{LAN}^{* *}$ & $\begin{array}{c}0.158 b \\
( \pm 0.022)\end{array}$ & $\begin{array}{c}0.142 \mathrm{a} \\
( \pm 0.011)\end{array}$ & $\begin{array}{c}0.142 \mathrm{a} \\
( \pm 0.012)\end{array}$ & $\begin{array}{l}0.155 \mathrm{ab} \\
( \pm 0.012)\end{array}$ & $\begin{array}{c}0.154 \\
( \pm 0.021)\end{array}$ \\
\hline \multicolumn{6}{|l|}{ Ala anterior } \\
\hline LAA* & $\begin{array}{c}0.406 a \\
( \pm 0.026)\end{array}$ & $\begin{array}{c}0.411 \mathrm{a} \\
( \pm 0.027)\end{array}$ & $\begin{array}{c}0.408 \mathrm{a} \\
( \pm 0.010)\end{array}$ & $\begin{array}{c}0.434 b \\
( \pm 0.017)\end{array}$ & $\begin{array}{c}0.408 \\
( \pm 0.026)\end{array}$ \\
\hline AAA (NS) & $\begin{array}{l}0.192 \mathrm{ab} \\
( \pm 0.011)\end{array}$ & $\begin{array}{l}0.192 \mathrm{ab} \\
( \pm 0.012)\end{array}$ & $\begin{array}{c}0.187 \mathrm{a} \\
( \pm 0.006)\end{array}$ & $\begin{array}{c}0.198 b \\
( \pm 0.013)\end{array}$ & $\begin{array}{c}0.192 \\
( \pm 0.012)\end{array}$ \\
\hline $\operatorname{LSM}^{* *}$ & $\begin{array}{c}0.037 \mathrm{~b} \\
( \pm 0.004)\end{array}$ & $\begin{array}{c}0.040 \mathrm{~b} \\
( \pm 0.005)\end{array}$ & $\begin{array}{c}0.039 \mathrm{~b} \\
( \pm 0.003)\end{array}$ & $\begin{array}{c}0.029 a \\
( \pm 0.004)\end{array}$ & $\begin{array}{c}0.038 \\
( \pm 0.005)\end{array}$ \\
\hline LVE (NS) & $\begin{array}{l}0.038 \mathrm{ab} \\
( \pm 0.005)\end{array}$ & $\begin{array}{c}0.039 \mathrm{~b} \\
( \pm 0.003)\end{array}$ & $\begin{array}{l}0.038 \mathrm{ab} \\
( \pm 0.003)\end{array}$ & $\begin{array}{c}0.035 a \\
( \pm 0.004)\end{array}$ & $\begin{array}{c}0.038 \\
( \pm 0.005)\end{array}$ \\
\hline
\end{tabular}




\begin{tabular}{lccccc}
\hline Variable & T. pretiosum & T. fuentesi & T. exiguum & T. pintoi & Media \\
\hline AVE** & $0.005 \mathrm{~b}$ & $0.006 \mathrm{~b}$ & $0.006 \mathrm{~b}$ & $0.004 \mathrm{a}$ & 0.005 \\
& $( \pm 0.001)$ & $( \pm 0.001)$ & $( \pm 0.001)$ & $( \pm 0.001)$ & $( \pm 0.001)$ \\
Ala posterior & & & & \\
LAP** & $0.177 \mathrm{~b}$ & $0.174 \mathrm{ab}$ & $0.168 \mathrm{a}$ & $0.217 \mathrm{c}$ & 0.177 \\
& $( \pm 0.011)$ & $( \pm 0.010)$ & $( \pm 0.009)$ & $( \pm 0.011)$ & $( \pm 0.014)$ \\
AAP (NS) & $0.032 \mathrm{ab}$ & $0.034 \mathrm{~b}$ & $0.031 \mathrm{a}$ & $0.032 \mathrm{ab}$ & 0.032 \\
& $( \pm 0.004)$ & $( \pm 0.004)$ & $( \pm 0.003)$ & $( \pm 0.004)$ & $( \pm 0.004)$ \\
LSM** & $0.050 \mathrm{~b}$ & $0.051 \mathrm{~b}$ & $0.045 \mathrm{a}$ & $0.048 \mathrm{ab}$ & 0.050 \\
& $( \pm 0.004)$ & $( \pm 0.004)$ & $( \pm 0.006)$ & $( \pm 0.006)$ & $( \pm 0.005)$ \\
\hline
\end{tabular}

Medias en hileras con letra diferente difieren: * Significativa, ** Altamente significativa. NS: No significativa.

Al realizar un análisis de grupos (análisis de cluster jerárquico) por el método de la distancia euclidiana al cuadrado de las diecisiete variables morfométricas referidas, se observó que solo seis presentaron información que permitieron diferenciar las especies determinadas por taxonomía tradicional. De este análisis, se obtuvieron los cuadros 3 y 4 en donde se presentan para cada variable, los grupos combinados y sus coeficientes. Los dendrogramas de cada variable se ilustran en las figuras 1 a 6 .

Considerando los valores máximos de los coeficientes de la distancia euclidiana al cuadrado de las estructuras referidas, se observó que los correspondientes a la longitud del proceso intervoselar de la genitalia y la longitud de la seta marginal del ala anterior presentaron menor valor (5.82 y 4.55 respectivamente), lo cual indica que los datos de las mediciones de estas variables fueron muy similares entre las muestras y presentaron una menor variación entre sus datos, en relación a los de las otras variables.

\section{DISCUSIÓN}

Un resumen de las relaciones entre las cuatro especies determinadas por morfología es: A) entre muestras con T. pretiosum (1)-(1), B) T. pretiosum con T. fuentesi (1)-(2), C) $T$. pretiosum con $T$. exiguum (1)-(3), D) $T$. pretiosum con $T$. pintoi (1)-(4), E) $T$. fuentesi con T. fuentesi (2)-(2), F) T. fuentesi con T. exiguum (2)-(3) y G) T. exiguum con T. pintoi (3)-(4). Estas relaciones reflejan la similaridad de las variables morfométricas que se presentan entre las especies. Se observó que las muestras de T. pretiosum presentaron una mayor cantidad de grupos combinados los cual es debido a que el número de muestra con esta especie fue 16, de T. fuentesi cuatro, $T$. exiguum dos y $T$. pintoi con una. 
García-González et al. Morfometría de Trichogramma

Cuadro 3. Grupos combinados de la distancia euclidiana al cuadrado (x 10-5) de seis variables morfométricas de Trichogramma de centros reproductores de México.

\begin{tabular}{|c|c|c|c|c|c|c|c|c|}
\hline \multicolumn{3}{|c|}{ LPIV } & \multicolumn{3}{|c|}{ LSF } & \multicolumn{3}{|c|}{ LVE } \\
\hline \multicolumn{3}{|c|}{ Grupo combinado } & \multicolumn{3}{|c|}{ Grupo combinado } & \multicolumn{3}{|c|}{ Grupo combinado } \\
\hline CA & CB & Co & $\mathbf{C A}$ & CB & Co & CA & $\mathbf{C B}$ & Co \\
\hline $17 \mathrm{M} 1(2)$ & 17M2(3) & 0.0 & 17M1(2) & $17 \mathrm{M} 2(3)$ & 0.0 & $13 \mathrm{M} 2(3)$ & $9 \mathrm{M} 1(4)$ & 0.0 \\
\hline $8(2)$ & 17M1(2) & 0.0 & $8(2)$ & $10(2)$ & 0.0 & $7(1)$ & 13M2(3) & 0.0 \\
\hline $2(1)$ & $10(2)$ & 0.0 & 11(1) & $4(2)$ & 0.0 & 13M1(1) & 17M1(2) & 0.0 \\
\hline 17M4(1) & $4(2)$ & 0.0 & $6(1)$ & 17M3(1) & 0.0 & $8(2)$ & $10(2)$ & 0.0 \\
\hline $1(1)$ & 17M4(1) & 0.0 & 9M2(1) & $14(1)$ & 0.0 & $4(2)$ & $8(2)$ & 0.0 \\
\hline $5 \mathrm{M} 2(1)$ & 17M3(1) & 0.0 & $12(1)$ & $5 \mathrm{M} 2(1)$ & 0.0 & $16(1)$ & 17M4(1) & 0.0 \\
\hline $15(1)$ & $16(1)$ & 0.0 & $1(1)$ & $12(1)$ & 0.0 & $7(1)$ & $16(1)$ & 0.0 \\
\hline $1(1)$ & $15(1)$ & 0.0 & $1(1)$ & $7(1$ & 0.0 & $11(1)$ & $15(1)$ & 0.0 \\
\hline 11(1) & $14(1)$ & 0.0 & $16(1)$ & 17M4(1) & 0.1 & 9M2(1) & $14(1)$ & 0.0 \\
\hline $6(1)$ & $5 \mathrm{M} 2(1)$ & 0.0 & $6(1)$ & $15(1)$ & 0.1 & $6(1)$ & $12(1)$ & 0.0 \\
\hline $7(1)$ & $12(1)$ & 0.0 & 9M2(1) & 11(1) & 0.1 & $7(1)$ & $11(1)$ & 0.0 \\
\hline $1(1)$ & $11(1)$ & 0.0 & $2(1)$ & $5 \mathrm{M} 1(1)$ & 0.1 & $5 \mathrm{M} 2(1)$ & 17M2(3) & 0.1 \\
\hline $3(1)$ & $9 \mathrm{M} 2(1)$ & 0.0 & $1(1)$ & 9M2(1) & 0.25 & $9 \mathrm{M} 2(1)$ & $4(2)$ & 0.1 \\
\hline $6(1)$ & $7(1)$ & 0.0 & $13 \mathrm{M} 1(2)$ & $8(2)$ & 0.9 & $6(1)$ & 13M1(1) & 0.1 \\
\hline $1(1)$ & $3(1)$ & 0.0 & $1(1)$ & $16(1)$ & 1.15 & $3(1)$ & 5M1(1) & 0.1 \\
\hline $1(1)$ & $6(1)$ & 0.1 & $2(1)$ & $3(1)$ & 1.25 & $6(1)$ & $7(1)$ & 0.2 \\
\hline 13M1(1) & $13 \mathrm{M} 2(3)$ & 0.1 & $13 \mathrm{M} 2(3)$ & 9M1(4) & 1.6 & $3(1)$ & $5 \mathrm{M} 2(1)$ & 0.45 \\
\hline $2(1)$ & $8(2)$ & 0.1 & 13M1(1) & 17M1(2) & 3.8 & $6(1)$ & $9 \mathrm{M} 2(1)$ & 1.03 \\
\hline $1(1)$ & 5M1(1) & 0.21 & $1(1)$ & $6(1)$ & 4.39 & $2(1)$ & $3(1)$ & 1.35 \\
\hline $2(1)$ & 13M1(1) & 0.41 & $1(1)$ & $2(1)$ & 8.81 & $2(1)$ & $6(1)$ & 3.74 \\
\hline $1(1)$ & $2(1)$ & 1.42 & 13M1(1) & $13 \mathrm{M} 2(3)$ & 25.1 & $1(1)$ & $2(1)$ & 13.16 \\
\hline $1(1)$ & 9M1(4) & 5.82 & $1(1)$ & $13 \mathrm{M} 1(1)$ & 105.3 & & & \\
\hline
\end{tabular}

LPIV: longitud del proceso intervoselar; LSF: longitud de la seta funicular; LVE: longitud de la vena estigmal; M: muestra: CA: grupo A; CB: grupo B; Co: coeficiente; (1) T. pretiosum; (2): T. fuentesi; (3): T. exiguum; (4): T. pintoi. 
Cuadro 4. Grupos combinados de la distancia euclidiana al cuadrado (x 10-5) de seis variables morfométricas de Trichogramma de centros reproductores de México (continuación).

\begin{tabular}{|c|c|c|c|c|c|c|c|c|}
\hline \multicolumn{3}{|c|}{ ALD } & \multicolumn{3}{|c|}{ LSMAA } & \multicolumn{3}{|c|}{ LPE } \\
\hline \multicolumn{3}{|c|}{ Grupo combinado } & \multicolumn{3}{|c|}{ Grupo combinado } & \multicolumn{3}{|c|}{ Grupo combinado } \\
\hline$\overline{\mathrm{CA}}$ & $\mathbf{C B}$ & Co & $\mathbf{C A}$ & $\mathbf{C B}$ & Co & $\mathbf{C A}$ & $\mathbf{C B}$ & Co \\
\hline $10(2)$ & $17 \mathrm{M} 2(3)$ & 0.0 & $8(2)$ & $17 \mathrm{M} 2(3)$ & 0.0 & $6(1)$ & $13 \mathrm{M} 2(3)$ & 0.0 \\
\hline $7(1)$ & $13 \mathrm{M} 1(3)$ & 0.0 & $3(1)$ & $13 \mathrm{M} 2(3)$ & 0.0 & $4(2)$ & 17M1(2) & 0.0 \\
\hline $13 \mathrm{M} 1(1)$ & $10(2)$ & 0.0 & $14(1)$ & $17 \mathrm{M} 1(2)$ & 0.0 & 17M3(1) & $8(2)$ & 0.0 \\
\hline $13 \mathrm{M} 1(1)$ & $8(2)$ & 0.0 & $4(2)$ & $10(2)$ & 0.0 & $12(1)$ & $17 \mathrm{M} 3(1)$ & 0.0 \\
\hline $5 \mathrm{M} 1(1)$ & $4(2)$ & 0.0 & $12(1)$ & $8(2)$ & 0.0 & $1(1)$ & $16(1)$ & 0.0 \\
\hline 17M3(1) & 17M4(1) & 0.0 & $15(1)$ & $16(1)$ & 0.0 & $9 \mathrm{M} 2(1)$ & $15(1)$ & 0.0 \\
\hline $9 \mathrm{M} 2(1)$ & 17M3(1) & 0.0 & $7(1)$ & $15(1)$ & 0.0 & $3(1)$ & 11(1) & 0.0 \\
\hline $5 \mathrm{M} 2(1)$ & $16(1)$ & 0.0 & 9M2(1) & $14(1)$ & 0.0 & $2(1)$ & $3(1)$ & 0.0 \\
\hline $9 \mathrm{M} 2(1)$ & $15(1)$ & 0.0 & $9 \mathrm{M} 2(1)$ & $5 \mathrm{M} 2(1)$ & 0.0 & $7(1)$ & 17M2(3) & 0.1 \\
\hline $6(1)$ & $14(1)$ & 0.0 & $11(1)$ & 13M1(1) & 0.0 & $12(1)$ & $10(2)$ & 0.1 \\
\hline $1(1)$ & $5 \mathrm{M} 2(1)$ & 0.0 & $2(1)$ & 11(1) & 0.0 & $1(1)$ & 17M4(1) & 0.1 \\
\hline $11(1)$ & $12(1)$ & 0.0 & $1(1)$ & $3(1)$ & 0.0 & $2(1)$ & $9 \mathrm{M} 2(1)$ & 0.1 \\
\hline $1(1)$ & 11(1) & 0.0 & $1(1)$ & $4(2)$ & 0.1 & $1(1)$ & $6(1)$ & 0.2 \\
\hline $2(1)$ & $3(1)$ & 0.0 & $7(1)$ & 17M4(1) & 0.1 & $7(1)$ & 13M1(1) & 0.25 \\
\hline 13M1(1) & $17 \mathrm{M} 1(2)$ & 0.1 & $2(1)$ & $12(1)$ & 0.1 & $5 \mathrm{M} 1(1)$ & $16(1)$ & 0.4 \\
\hline $1(1)$ & 9M2(1) & 0.1 & $5 \mathrm{M} 1(1)$ & $7(1)$ & 0.18 & $1(1)$ & $12(1)$ & 0.68 \\
\hline $5 \mathrm{M} 1(1)$ & $7(1)$ & 0.1 & $2(1)$ & 9M2(1) & 0.25 & $7(1)$ & $4(2)$ & 1.67 \\
\hline $2(1)$ & 13M1(1) & 0.16 & $1(1)$ & $6(2)$ & 0.28 & $2(1)$ & $14(1)$ & 2.14 \\
\hline $1(1)$ & $6(1)$ & 0.27 & $1(1)$ & $2(1)$ & 1.54 & $1(1)$ & $2(1)$ & 3.29 \\
\hline $1(1)$ & 5M1(1) & 0.58 & $5 \mathrm{M} 1(1)$ & 9M1(4) & 2.54 & $1(1)$ & $7(1)$ & 12.15 \\
\hline $1(1)$ & $2(1)$ & 2.68 & $1(1)$ & 5M1(1) & 4.55 & $1(1)$ & 5M1(1) & 24.23 \\
\hline $1(1)$ & 9M1(4) & 7.80 & & & & $1(1)$ & 9M1(4) & 174.7 \\
\hline
\end{tabular}

ALD: ancho de la lámina dorsal; LSMAA: longitud de la seta marginal del ala anterior; LAP: longitud del ala posterior; M: muestra: CA: grupo A; CB: grupo B; Co: coeficiente; (1) T. pretiosum; (2): T. fuentesi;

(3): T. exiguum; (4): T. pintoi. 
Los dendrogramas (Figs. 1 a 6), permitieron ubicar los diferentes grupos que el análisis de grupos determinó en base a la similaridad de las mediciones de cada estructura de cada centro y de cada especie (indicada entre paréntesis). De estos dendrogramas se puede inferir que el correspondiente a la longitud del proceso intervoselar de la genitalia (Fig. 1), permitió ubicar tres grupos. En el grupo A se observan los dos centros de T. exiguum (13M2 y 17M2) y tres de los cuatro centros de T. fuentesi ( 8,10 y 17M1), la muestra restante de esta especie (4) se ubicó en el grupo B. En el mismo grupo A se ubicaron dos centros de T. pretiosum (2 y $13 \mathrm{M} 1$ ) los cuales forman los grupos combinados 2(1)-8(2) y 13M1(1)-13M2, ambos con un coeficiente de 0.10 (x 10-5). En el grupo B se ubicaron 14 de los dieciséis centros de $T$. pretiosum, en tanto que en el grupo C se ubicó el centro 9M1 de T. pintoi. De manera general se puede indicar que esta estructura permitió ubicar mejor las cuatro especies referidas, aunque algunos centros de estas especies, en particular de $T$. fuentesi y $T$. pretiosum tuvieron una alta similaridad de sus datos entre ellas.

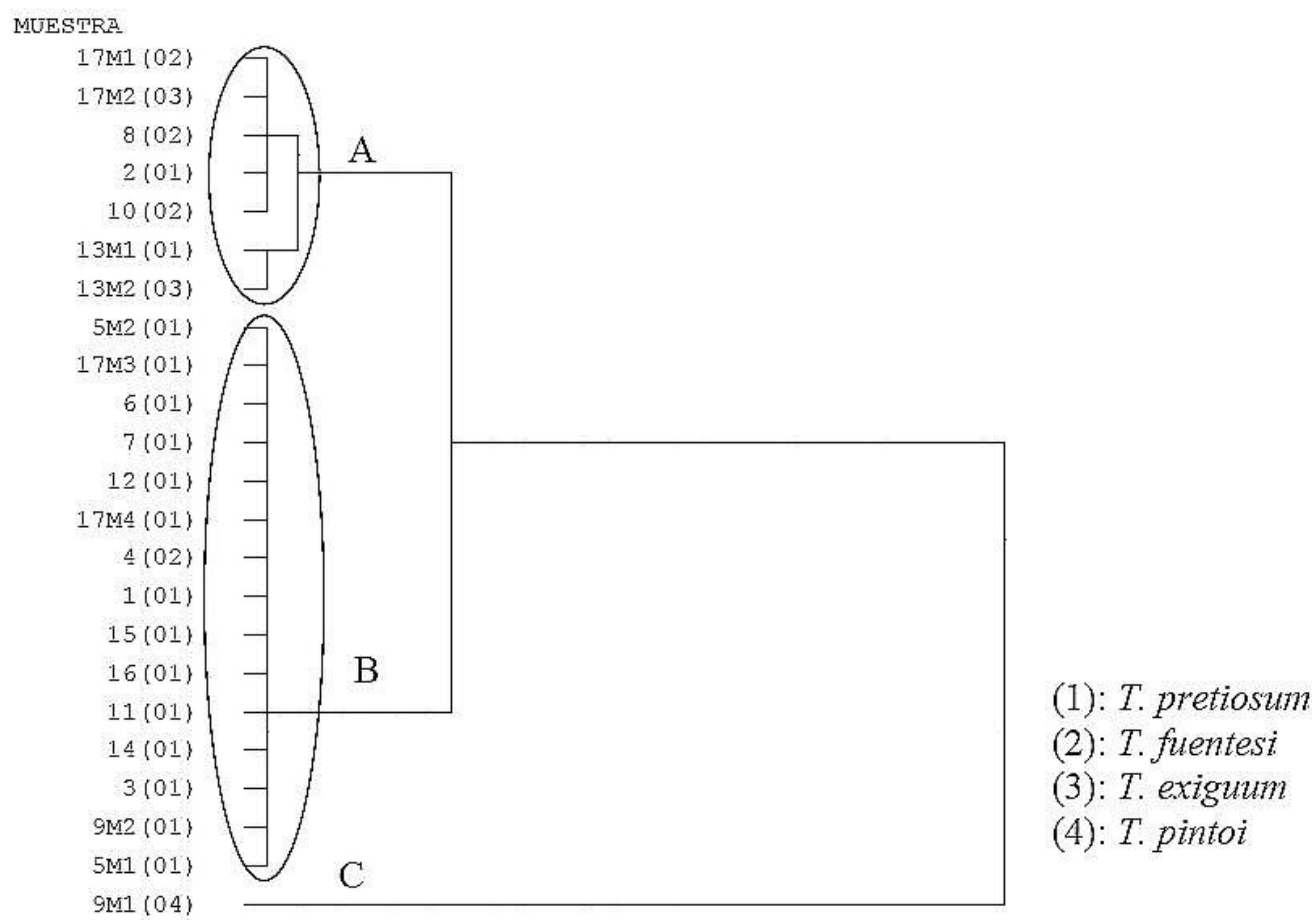

Figura 1. Dendograma de la longitud del proceso intrevoselar de la genitalia de cuatro especies de Trichogramma de centros reproductores de México. 
En el dendrograma de la longitud de la seta funicular de la antena (Fig. 2), se ubican claramente dos grupos. El grupo A incluye a las especies con setas funiculares cortas a excepción del centro 4 de T. fuentesi, el cual forma el grupo combinado 11(1)-4(2). Este grupo es formado por los dos centros de T. exiguum (13M2 y 17M2), el centro 9M1 de $T$. pintoi y tres de los cuatro centros de $T$. fuentesi. En este grupo también se presenta el centro 13M1 de T. pretiosum, el cual forma el grupo combinado 13M1(1)-8(2) con un coeficiente de 0.90 (x 10-5). En el grupo B se incluyen quince de los dieciséis centros de T. pretiosum el cual es una de las especies de Trichogramma que se caracteriza por tener setas funiculares largas. Dentro de este grupo se pueden ubicar tres subgrupos: uno formado por los centros $2,5 \mathrm{M} 1$ y $3 ; 6,17 \mathrm{M} 3$ y 15 ; y el tercer grupo con diez de los dieciséis centros que lo conforman.

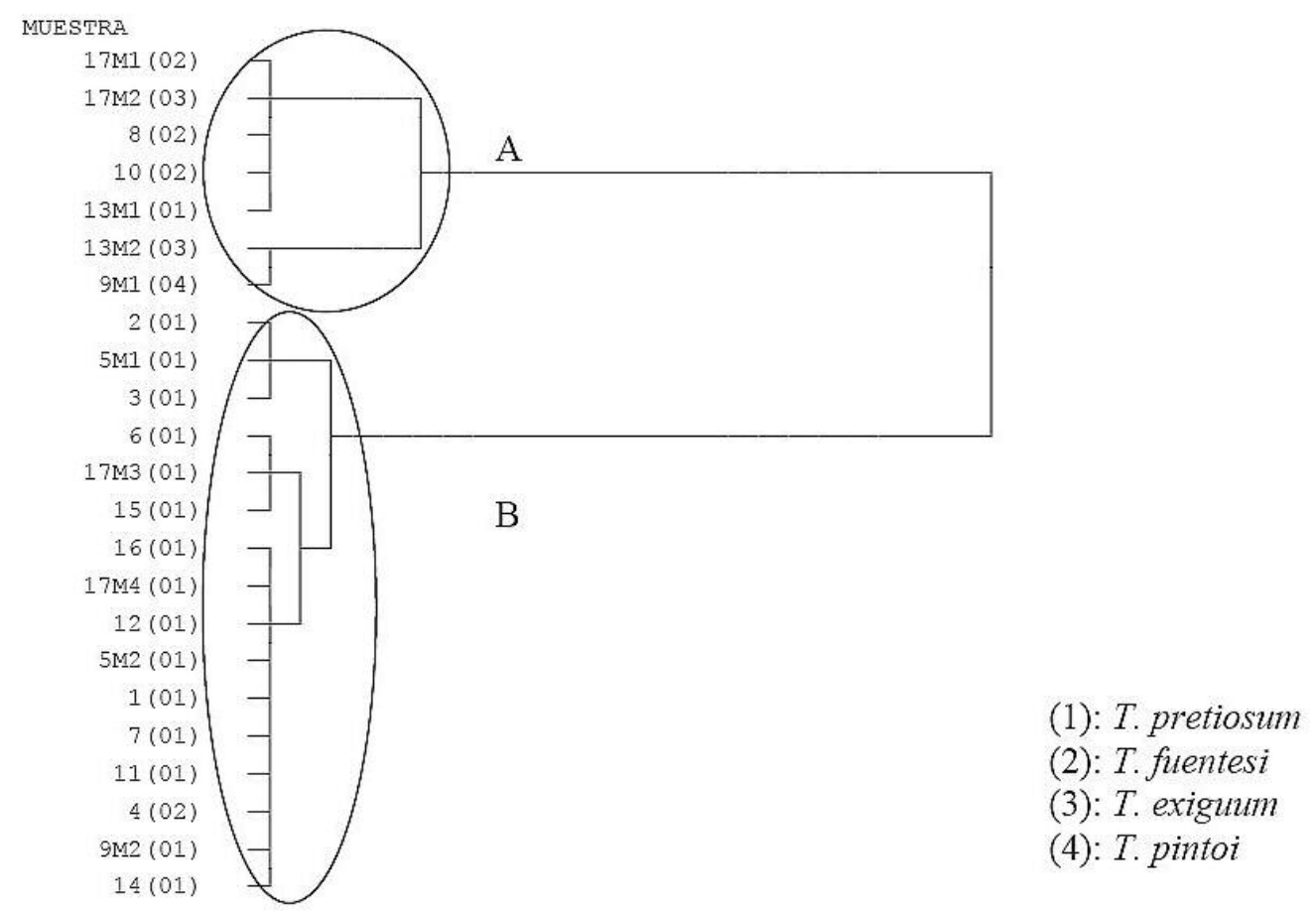

Figura 2. Dendograma de la longitud de la seta funicular de la antena de cuatro especies de Trichogramma de centros reproductores de México. 
En la figura 3 correspondiente al dendrograma de la longitud de la vena estigmal del ala anterior, pueden apreciarse claramente tres grupos y en forma independiente a los centros 1 y 2 , sin embargo el segundo grupo incluye a los cuatro centros de T. fuentesi (4, 8, 10 y $17 \mathrm{M} 1)$, en tanto en los dos grupos restantes se encuentran centros de $T$. pretiosum, $T$. exiguum y el centro de T. pintoi. Los centros de T. pretiosum $9 \mathrm{M} 2$ y 14 también se ubicaron en este grupo las cuales formaron los grupos combinados 9M2(1)-14(1) y 9M2(1)-4(2) con coeficientes de 0.00 y 0.10 (x 10-5). Se puede inferir que esta estructura permitió ubicar en un solo grupo los centros de T. fuentesi de las demás especies referidas. Los dendrogramas correspondientes al ancho de la lamina dorsal de la genitalia, longitud de la seta marginal del ala anterior y longitud del ala posterior (Fig. 4,5,6) permitieron separar a $T$. pintoi de T. pretiosum, $T$. fuentesi y T. exiguum.

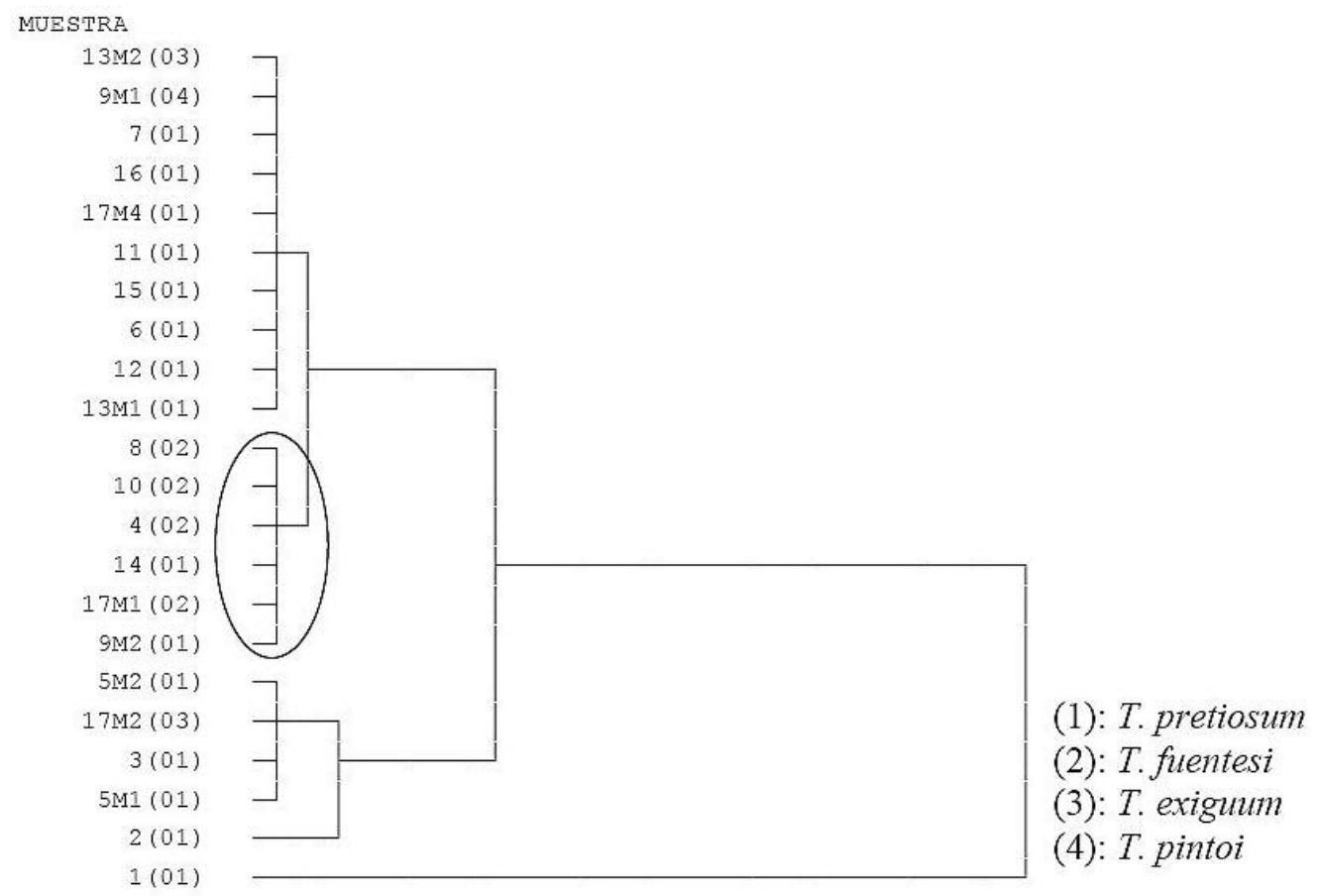

Figura 3. Dendograma de la longitud de la vena estigmal del ala anterior de cuatro especies de Trichogramma de centros reproductores de México. 


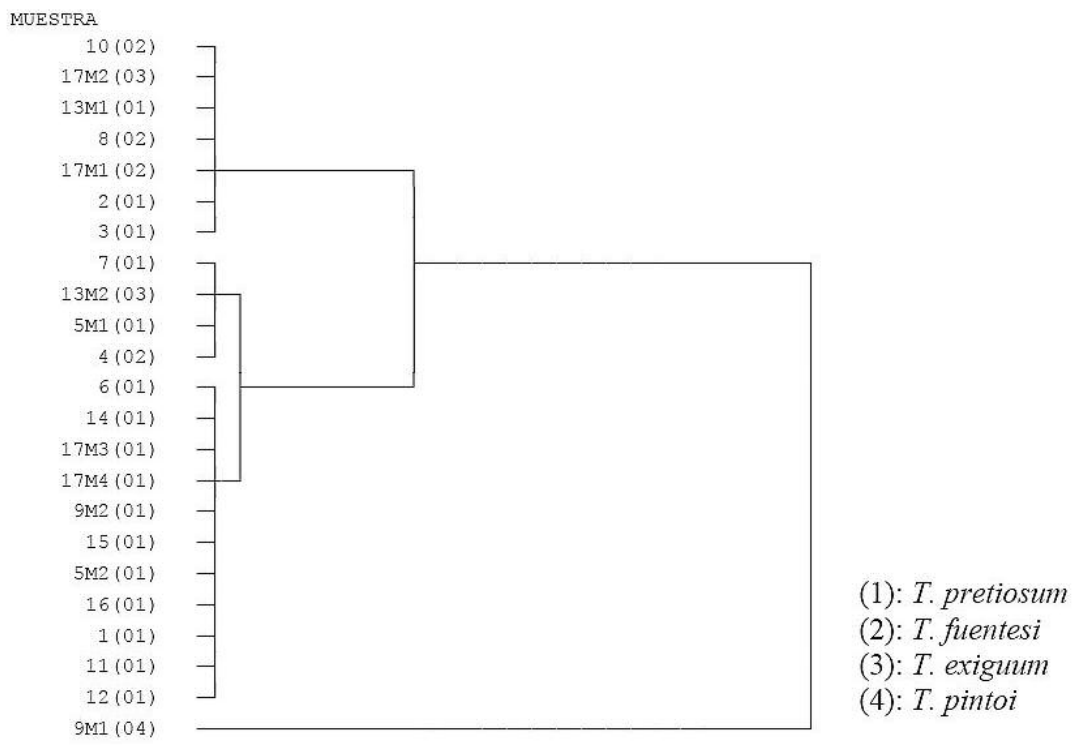

Figura 4. Dendograma del ancho de la lámina dorsal de la genitalia de cuatro especies de Trichogramma de centros reproductores de México.

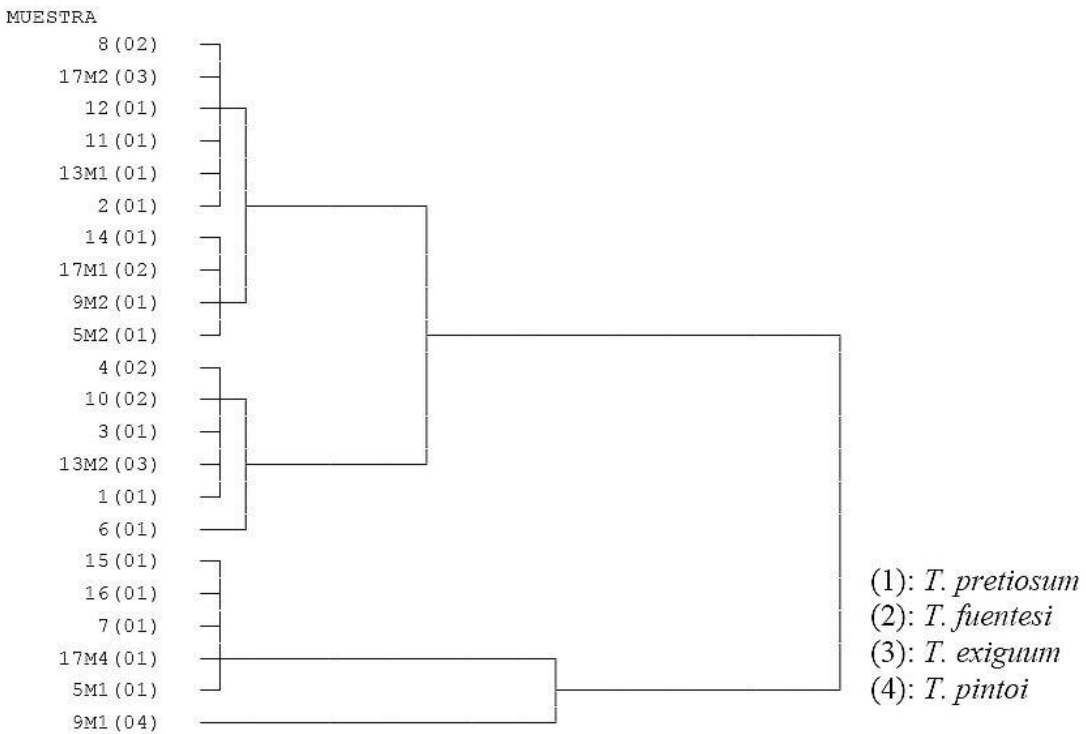

Figura 5. Dendograma de la longitud de seta marginal del ala anterior de cuatro especies de Trichogramma de centros reproductores de México. 


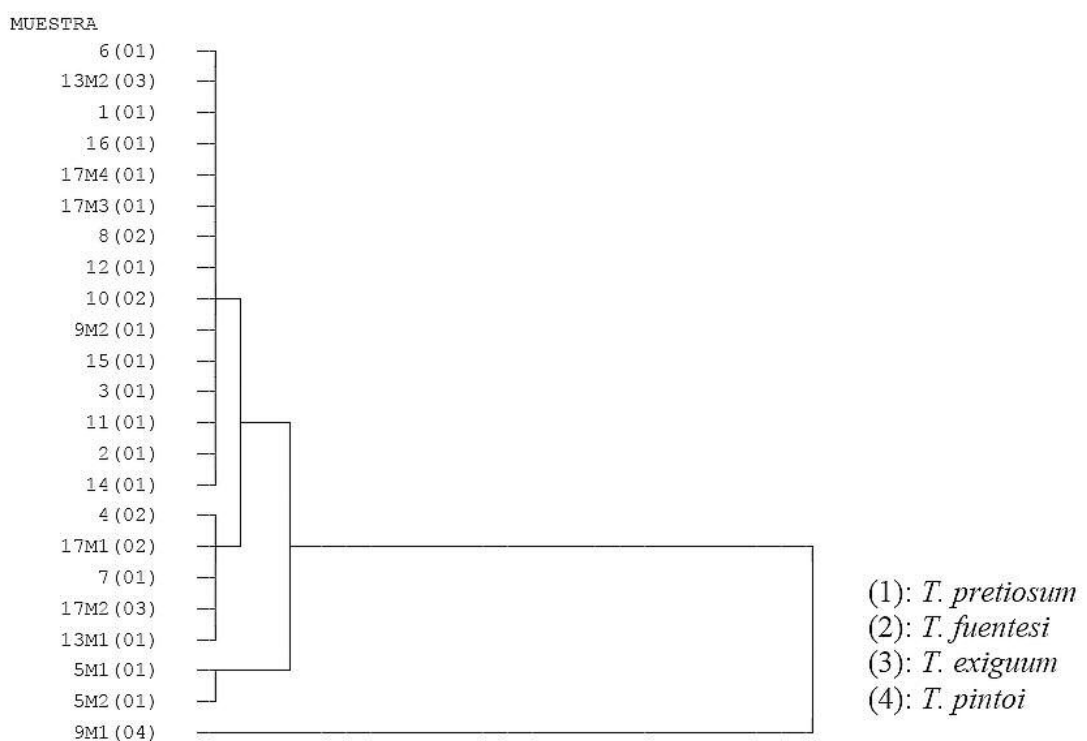

Figura 6. Dendograma de la longitud del ala posterior de cuatro especies de Trichogramma de centros reproductores de México.

Además de la taxonomía basada en caracteres morfológicos, actualmente existen varias técnicas que han sido evaluadas para diferenciar especies de Trichogramma, entre estas se tiene a las técnicas basadas en: diferenciación enzimática, reproducción ínterespecifica, moleculares y morfométricas. Una limitante de la taxonomía tradicional basada en caracteres morfológicos en la identificación de especies de Trichogramma, es que esta desarrollada en la morfología de los machos, lo cual restringe la determinación de especimenes colectados en campo, los cuales en un gran número de casos sólo se colectan hembras y de poblaciones teletoquias en las cuales sólo se tienen hembras. De las técnicas evaluadas recientemente, las moleculares y enzimáticas tienen la ventaja de diferenciar tanto hembras como machos, sin embargo para poder realizarlas es necesario estar bien capacitado y contar con equipos y suministros muy costosos.

La morfometría tiene la facultad de poder corroborar o confirmar especies de Trichogramma que previamente hayan sido determinadas en base a características morfológicas, principalmente en base a variables morfométricas de los machos, aunque Kostadinov y Pintureau (1991) diferenciaron tres especies de Trichogramma por morfometría de hembras. Una ventaja que tiene esta técnica con respecto a las técnicas moleculares es que no se requiere equipos y suministros muy costosos para poder desarrollarla. Sólo se requiere como equipo básico, un microscopio compuesto 
con foto tubo, una cámara fotográfica de alta resolución y equipo de cómputo con analizador de imágenes y Microsoft office ${ }^{\circledR}$.

En la actualidad existen pocos estudios de diferenciación de especies de Trichogramma por morfometría (Kostadinov y Pintureau, 1991; Galán y Rodríguez, 1993; Pintureau, 1993; Burks y Heraty, 2002;) y los que existen han considerado muy pocas especies reportadas en Norte América y en especifico para México entre las que se tienen a $T$. exiguum, $T$. fuentesi, $T$. pretiosum y $T$ pintoi.

Es importante considerar que la aplicación de la morfometría y de las técnicas moleculares debe enfocarse a especies que por taxonomía morfológica no pueden ser diferenciadas, esto es a especies sibilinas o hermanas (Pinto et al., 1992; Burks y Heraty, 2002), ya que un gran número de especies de Trichogramma pueden ser determinadas por taxonomía morfológica con precisión.

\section{CONCLUSIONES}

La longitud de la seta funicular de la antena (LSF), permitió separar a T. pretiosum y T. exiguum de T. fuentesi y T. pintoi; estas dos últimas son diferenciadas por su genitalia.

Con la longitud del proceso intervoselar (LPIV) fue posible separar a T. pretiosum y $T$. pintoi de $T$. fuentesi y $T$. exiguum, aunque estas dos última fueron estadísticamente iguales.

Las estructuras que presentaron resultados no significativos $(>0.05)$ para la separación de las especies fueron: el ancho de la ala anterior (AAA), longitud de la cápsula genital (LCG), la longitud de la vena estigmal de la ala anterior (LVE) y el ancho de la ala posterior (AAP).

El dendrograma de la longitud del proceso intervoselar de la genitalia (LPIV) permitió separar las cuatro especies, aunque las especies de algunos centros (2, 13M1 y 4), en particular de $T$. fuentesi y $T$. pretiosum, tuvieron una alta similaridad.

Con los dendrogramas correspondientes a la longitud del proceso intervoselar de la genitalia (LPIV), ancho de la lámina dorsal de la genitalia (ALD), longitud de la seta marginal del ala anterior (LSMAA) y longitud del ala posterior (LAP), fue posible separar a $T$. pintoi de $T$. pretiosum, T. fuentesi y T. exiguum.

En el dendrograma de la longitud de la vena estigmal del ala anterior, se separó a T. fuentesi de las demás especies referidas.

La morfometría es una buena técnica de diagnostico de especies de Trichogramma. Sus diagnósticos son menos costosos que los realizados por técnicas moleculares, sin embargo en la actualidad su aplicabilidad es limitada. 


\section{LITERATURA CITADA}

Anónimo. 1999a. Reference Guide Image Pro Plus Version 4.0 for Windows [CDROM]. Media Cybernetics. Silver Spring, MD.

Anónimo. 1999b. SPSS 10.0 for Windows. [CDROM]. Chicago, IL., USA.

Arredondo B.H. 1993. El control biológico y sus metodologías. In: Reyes E, Pérez PS. (eds.). Primer taller sobre control biológico de plagas agrícolas. Centro Nacional de Referencia de Control Biológico-DGSV Tecomán, Col. 20-28

Basso C, B. Pintureau \& G. Grille. 1999. Taxonomic study of two Trichogramma species from Uruguay (Hym.: Trichogrammatidae). Boletín de Sanidad Vegetal, Plagas, 25 (3): 373-382.

Burks R.A. \& J.M. Heraty. 2002. Morphometric analysis of four species of Trichogramma Westwood (Hymenoptera: Trichogrammatidae) attacking codling moth and other tortricid pests in North America. Journal Hymenoptera Research, 11(2):167-187.

Doutt R.L. \& G. Viggiani. 1968. The classification of the Trichogrammatidae (Hymenoptera: Chalcidoidea). Proceedings of the California Academy of Sciences, 35(20): 477-586.

Flanders S.E. 1930. Mass production of egg parasites of the genus Trichogramma. Hilgardia. 4: 465501.

Galán M. \& J. Rodríguez. 1993. Caracterización morfométrica de Trichogramma Westwood (Hymenoptera: Trichogrammatidae). Revista Protección Vegetal, 8:157-165.

González H.A. 2000. Chalcidoidea (Hymenoptera). Pp. 649-659. In: Biodiversidad, Taxonomía y Biogeografía de Artrópodos de México Vol. II, Lorete-Bousquets J., González-Soriano E. y Papayero N. (eds.). UNAM. México, D.F.

Kostadinov D. \& B. Pintureau. 1991. A possibility to discriminate females of three closely related species of Trichogramma (Hymenoptera: Trichogrammatidae) with special purpose analysis of the type of Trichogramma evanescens Westwood. Annales de la Société Entomologique de France, 27 (4): 393-400.

Moreno G.F. \& I.M. Pérez. 2002. El empleo de Trichogramma en control biológico de plagas: problemas taxonómicos. Boletín de la Sociedad Entomológica Aragonesa, 31: 239-242.

Owen K.A. \& J.D. Pinto. 2004. Pachamama, an uncommon and distinctive new genus of Trichogrammatidae (Hymenoptera: Chalcidoidea) from tropical America. Zootaxa. 664: 1-8.

Pinto J.D. 1997. Trichogrammatidae. Pp. 726-752. In: Annoted Key to The Genera of Nearctic Chalcidoidea (Hymenoptera), Gibson A, Huber J and Woolley J. (eds.). NRC Research Press. Ottawa, Ontario, Canada.

Pinto J.D. 1998. Systematics of the north american species of Trichogramma Westwood (Hymenoptera: Trichogrammatidae). Memoirs of the Entomological Society of Washington. 22: 1-287.

Pinto J.D. \& J. George. 2004. Kyuwia, a new genus of Trichogrammatidae (Hymenoptera) from Africa. Proceedings of the Entomological Society of Washington. 106 (3): 531-539.

Pinto J.D., D.J. Kazmer, G.R. Platner \& C.A Sassaman. 1992. Taxonomy of the Trichogramma minutum complex (Hymenoptera: Trichogrammatidae): allozymic variation and its relatioship to reproductive and geographic data. Annls of the Entomological Society of America, 85: 413-422.

Pinto J.D., E.R. Oatman \& G.R. Platner. 1983. The identity of two closely related and frequently encountered species of new world Trichogramma (Hymenoptera: Trichogrammatidae). Proccedings of the Entomological Society of Washington, 85(3): 588-593.

Pinto J.D., E.R. Oatman \& G.R. Platner. 1986. Trichogramma pretiosum and a new cryptic species occurring sympatrically in Southwestern North America (Hymenoptera: Trichogrammatidae). Annals of the Entomological Society of America, 79: 1019-1028. 
Pinto J.D. \& A. K. Owen. 2004. Adryas, a new genus of Trichogrammatidae (Hymenoptera: Chalcidoidea) from the New World Tropics. Proceedings of the Entomological Society of Washington. 106 (4): 905-922.

Pinto J.D., G.R. Platner \& C.A. Sassaman. 1993. Electrophoretic study of two closely species of North American Trichogramma: T. pretiosum and T. deion (Hymenoptera: Trichogrammatidae). Annls of the Entomological Society of America, 86 (6):702-709.

Pinto J.D., R. Stouthamer, G.R. Platner \& E.R. Oatman. 1991. Variation in reproductive compatibility in Trichogramma and its taxonomic significance (Hymenoptera: Trichogrammatidae). Annals of the Entomological Society of America. 84(1): 37-46.

Pintureau B. 1993. Morphometric analysis of the genus Trichogramma Westwood (Hymenoptera: Trichogrammatidae) in Europe. Canadian Entomologist, 125 (2): 367-378

Rodríguez-del-Bosque L.A. \& B.H.C. Arredondo. 1999. Quién es Quién en el control Biológico en México. INIFAP-CIRNE. Campo Experimental Río Bravo. Folleto Técnico Núm. 23 Tamaulipas, México. 147 p.

Van Lenteren, J. \& V.H. Bueno. 2003. Augmentative biological control of arthropods in Latin America. Biocontrol 48 (2): 123-139 